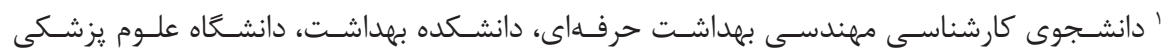

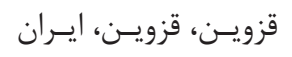

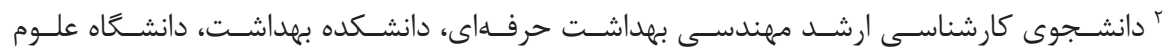

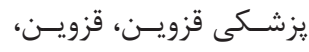

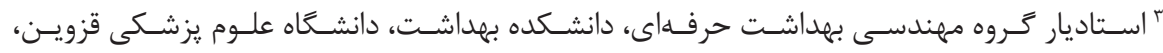

$$
\text { قزويـن، ايـران }
$$

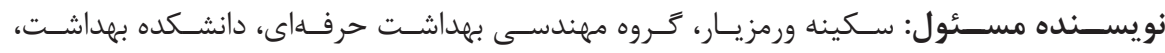

جكيده

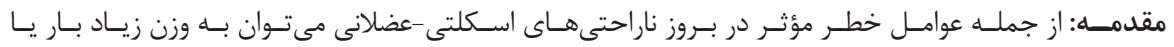

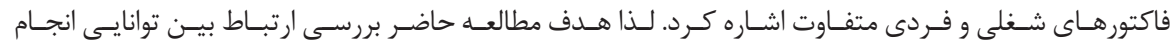

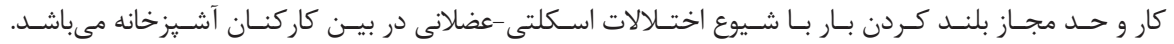

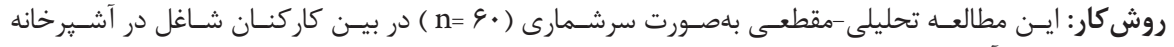

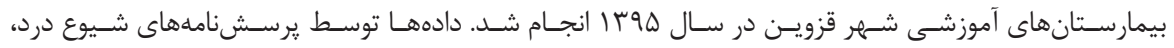

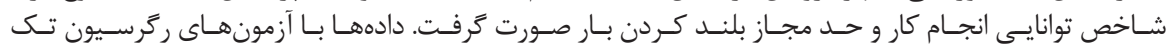

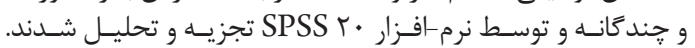

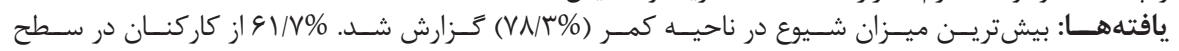

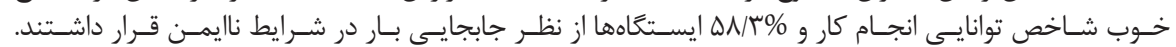

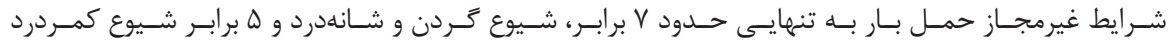

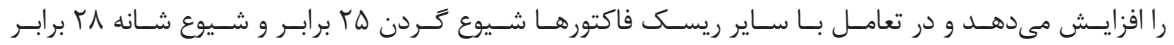

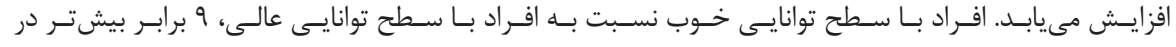

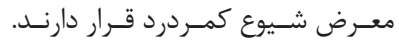

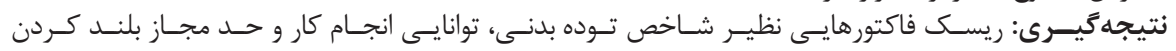

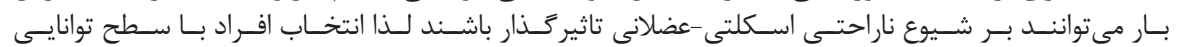

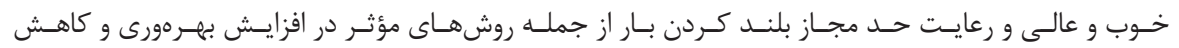

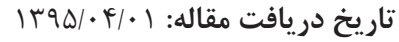

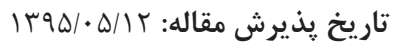

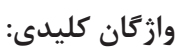
اختلالات اسكلتى -عضلانى كليدى شاخص توانايى انجام كار (WAI)

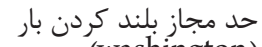
(washington)

همدامى حقفوق نشر براى دانشكاه علوم يزشكى

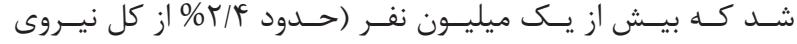

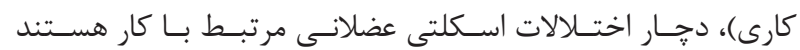

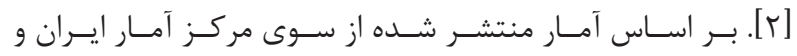

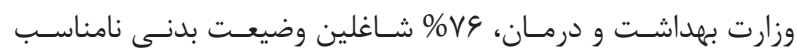

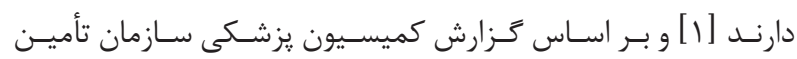

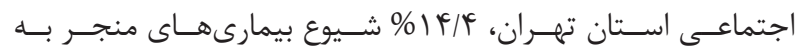

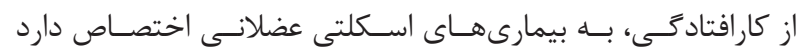

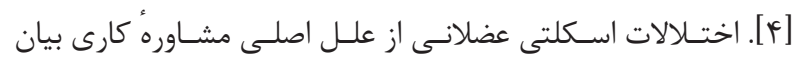

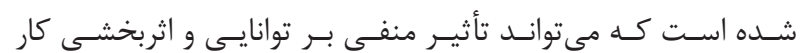

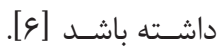

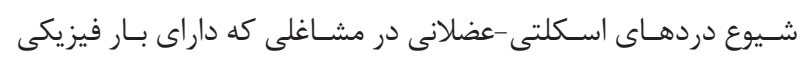

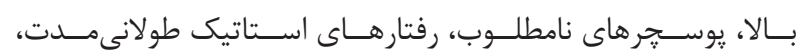

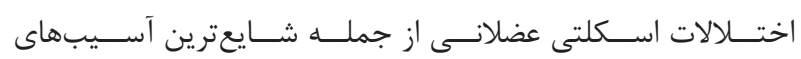

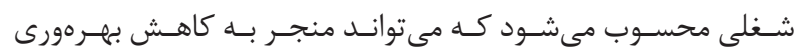

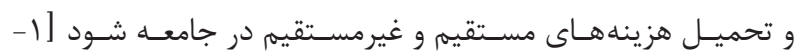

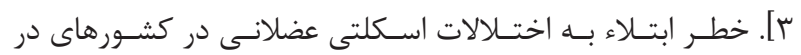

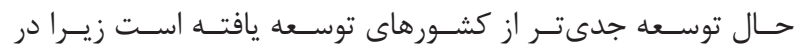

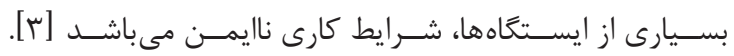

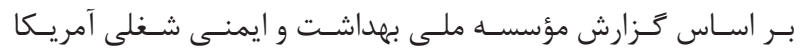
(NIOSH)

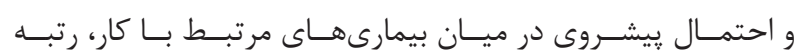

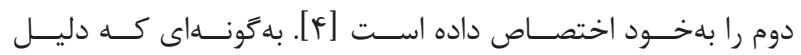

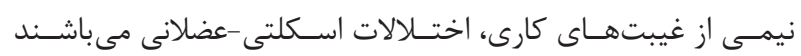

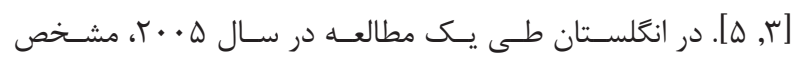




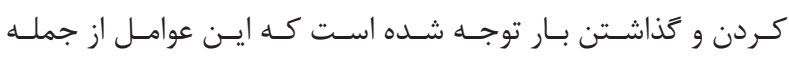

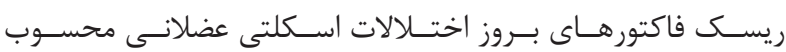

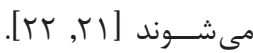

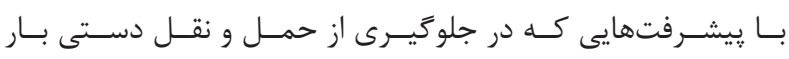

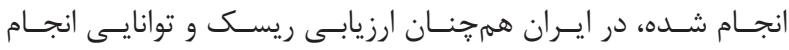

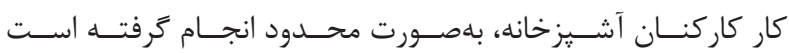

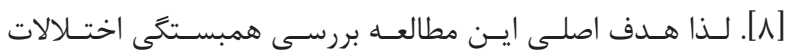

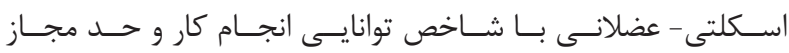

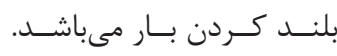

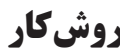

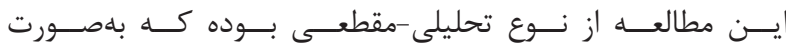

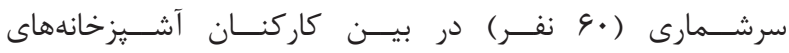

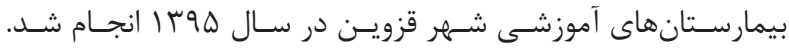

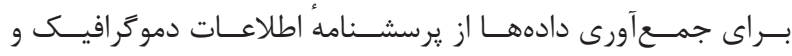

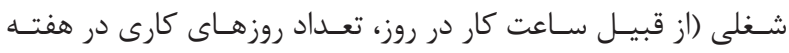

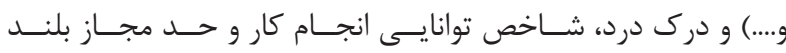

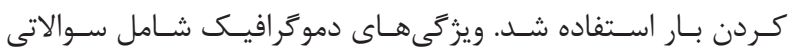

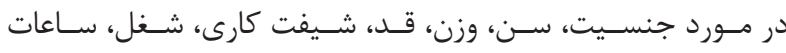

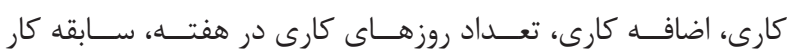

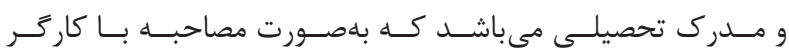
جمــعآورى گرديسـد.

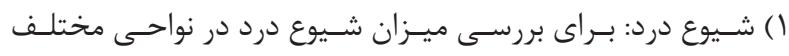

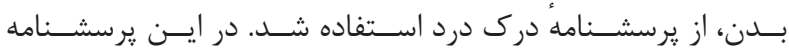

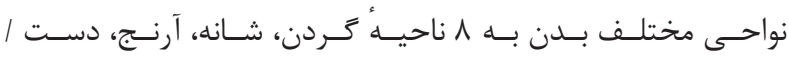

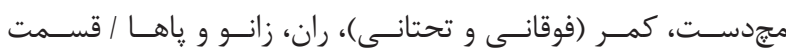

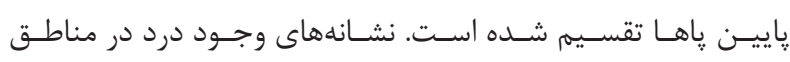

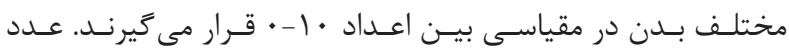

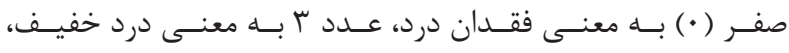

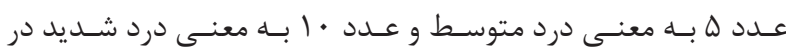

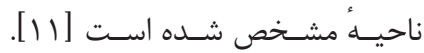

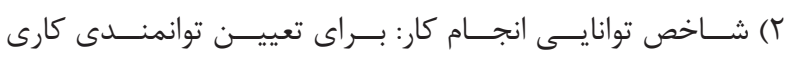

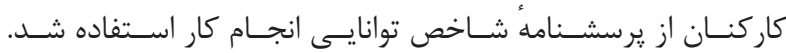

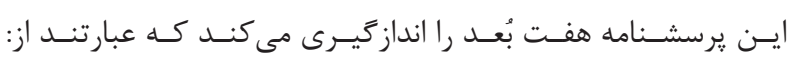

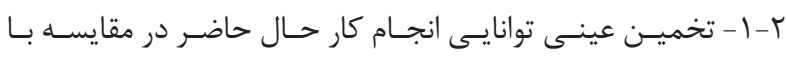

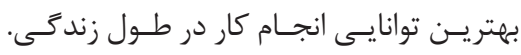

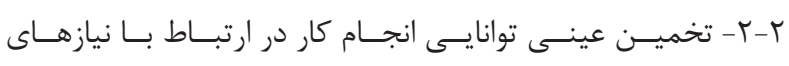

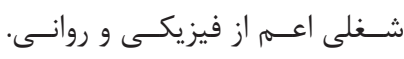
ץ-ץ- تعداد بيمارىهاى تشخيص داده شده.

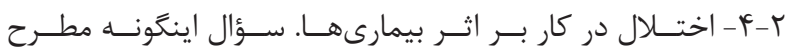

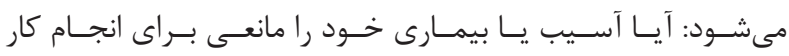

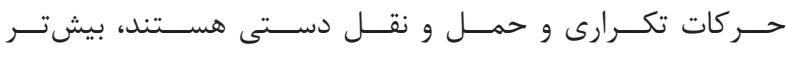

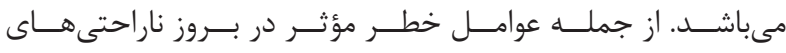

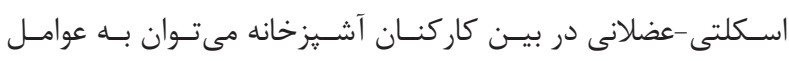

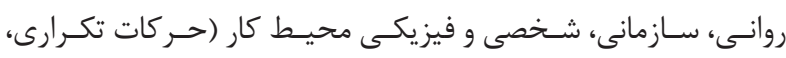

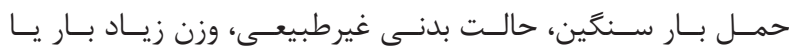

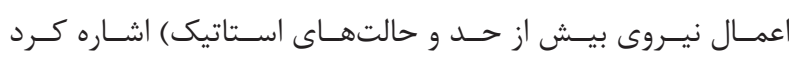

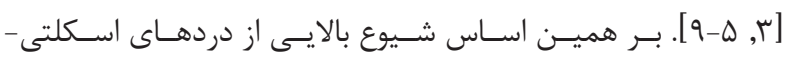

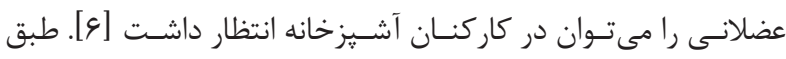

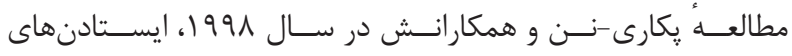

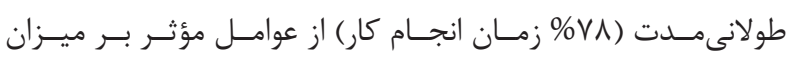

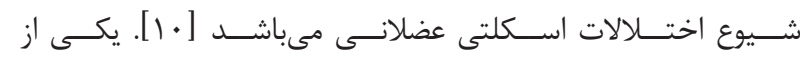

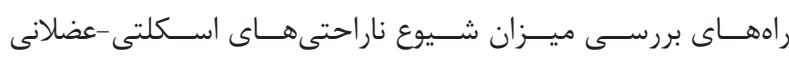

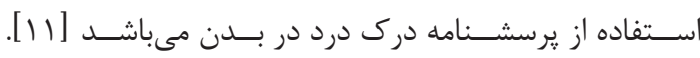

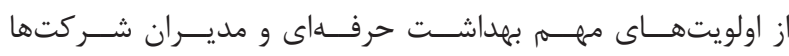

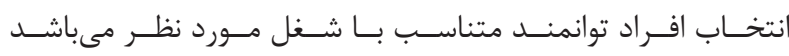

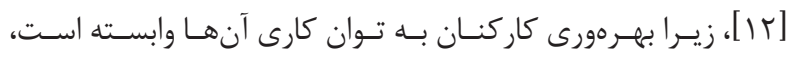

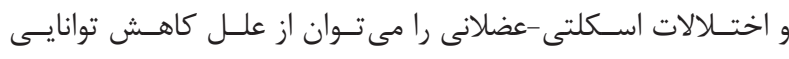

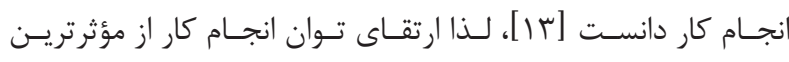

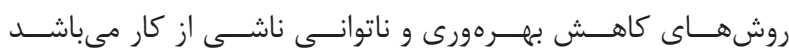

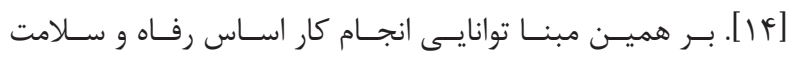

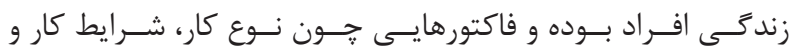

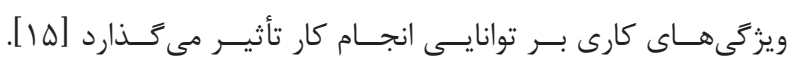

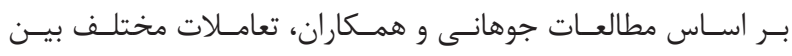

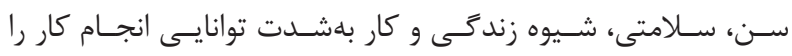

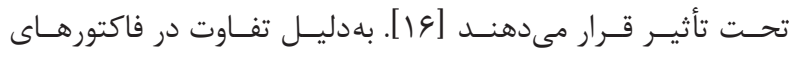

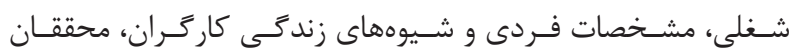

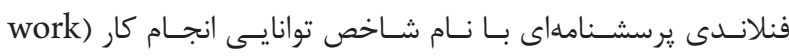
(ability index, WAI

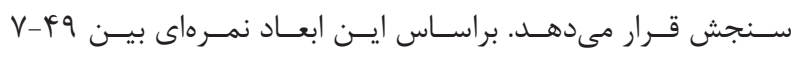

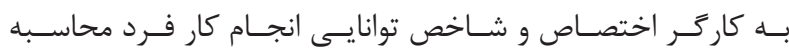

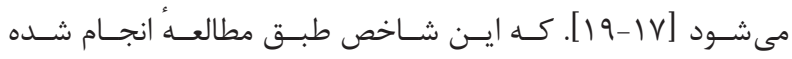

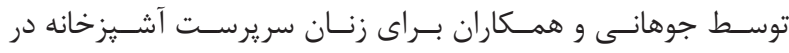

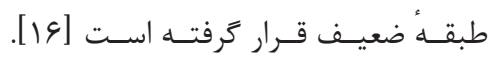

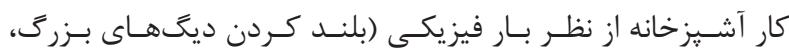

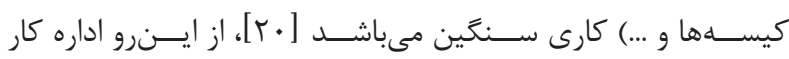

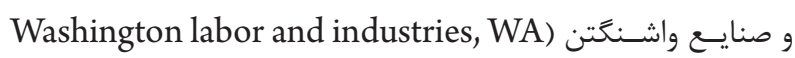
(L\&I

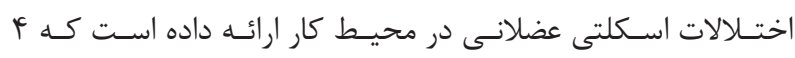

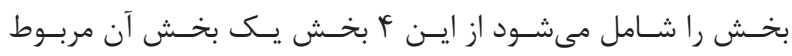

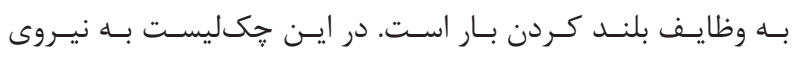

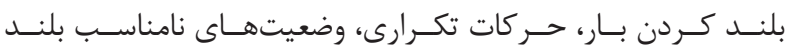




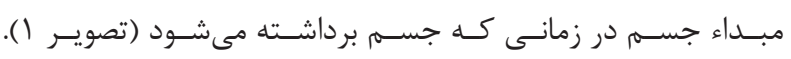

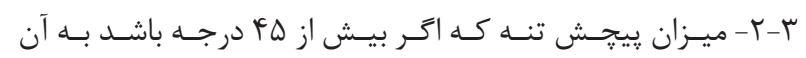

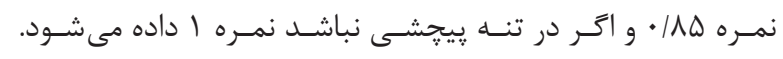

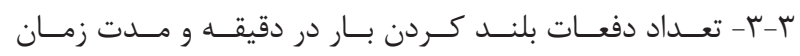

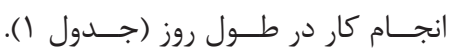

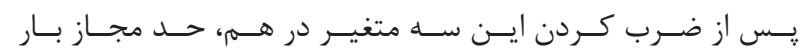

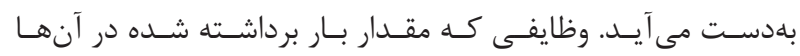

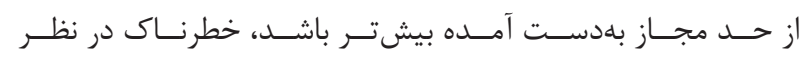

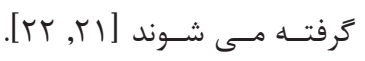

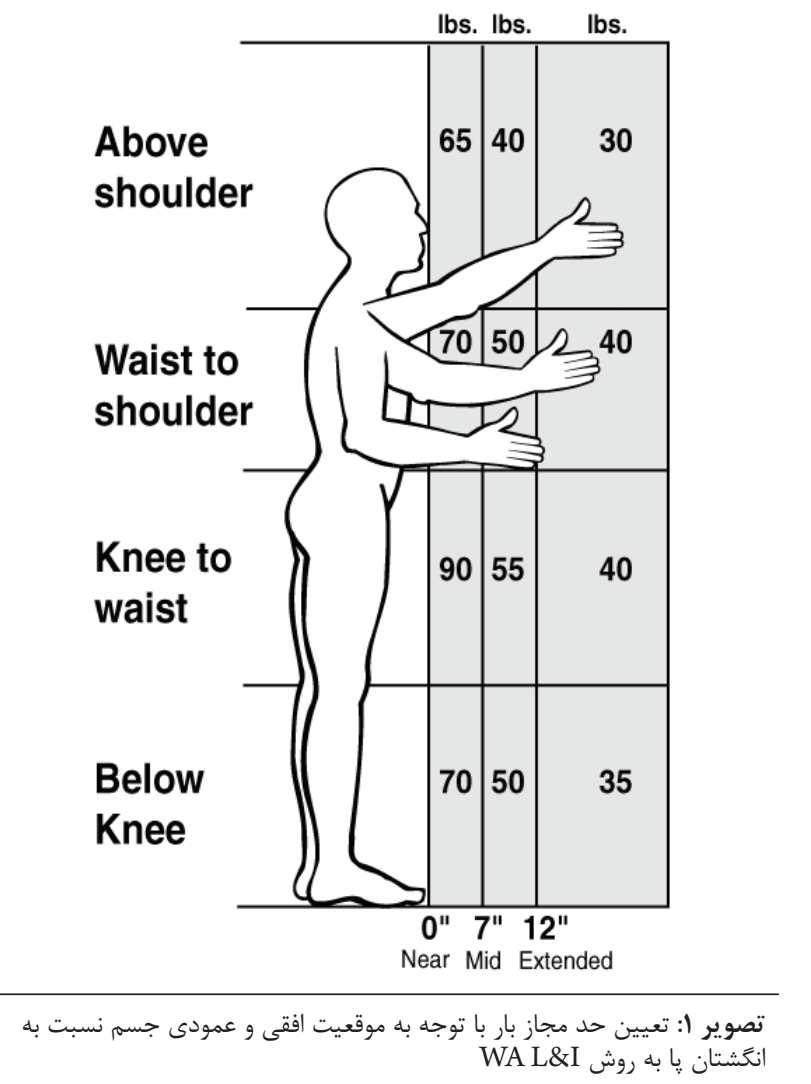

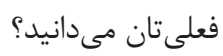

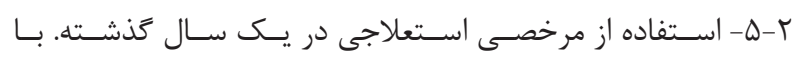

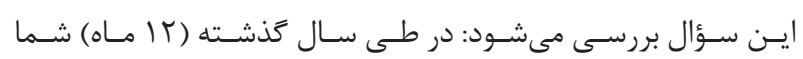

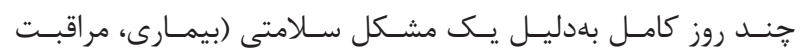

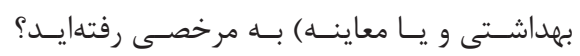

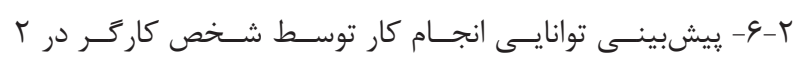
سـال آينـده.

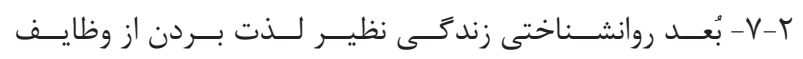

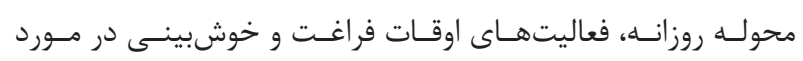

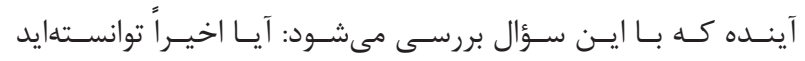

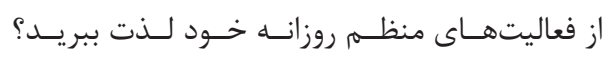

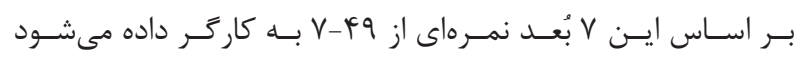

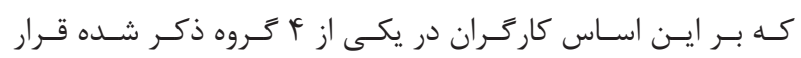

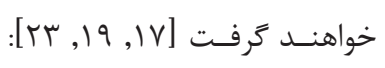

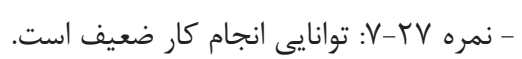

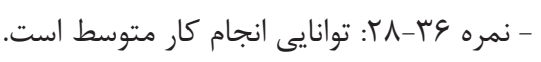

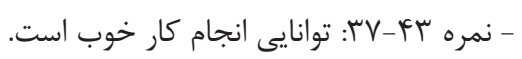

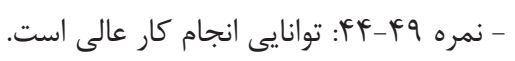

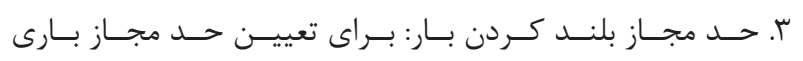

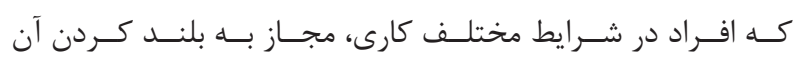

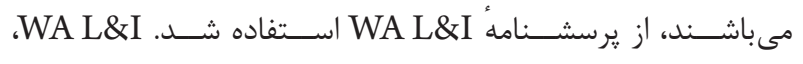

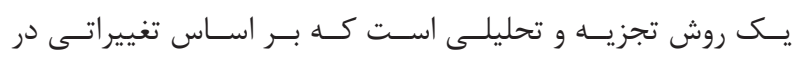

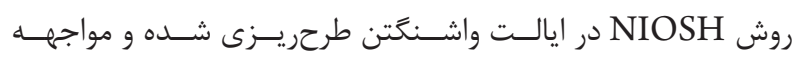

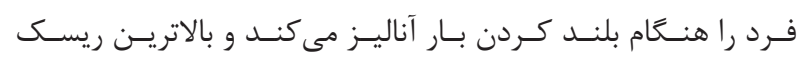

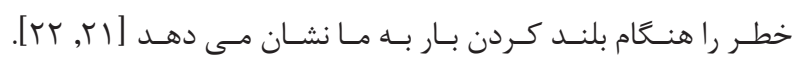

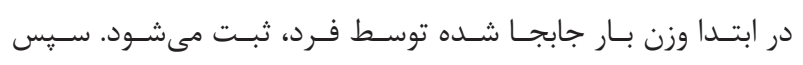

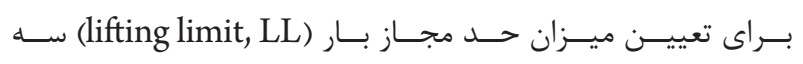

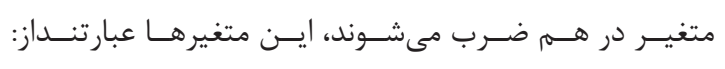

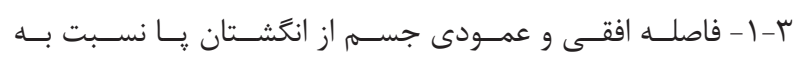

\begin{tabular}{|c|c|c|c|}
\hline \multicolumn{4}{|c|}{ جدول ا: تعيين نمره مربوط به بلند كردن جسم بر حسب تعداد دفعات بلند كردن بار و مدت زمان انجام اين كار به روش WA L\&I } \\
\hline \multicolumn{3}{|c|}{ جند ساعت در روز اين كار را انجام مى دهيد؟ } & جندبار در دقيقه جسم را بلند مىكنيد؟ \\
\hline بيشتر از Y ساعت & ا تا r ساعت & كمتر از ا ساعت & \\
\hline$\cdot / \wedge \Delta$ & .190 & 1 & ا بار در هر ه-r دقيقه \\
\hline$\cdot / V Q$ & $\cdot / 9$ & .190 & ا بار در هر دقيقه \\
\hline$\cdot 190$ & $\cdot / \Lambda \Delta$ & $\cdot 19$ & 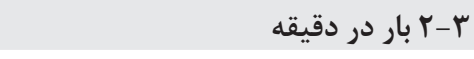 \\
\hline$\cdot / 4 \Delta$ & $\cdot / V$ & $\cdot / \Lambda \Delta$ & 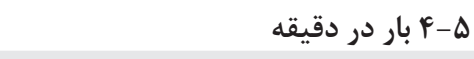 \\
\hline$\cdot / T \Delta$ & $\cdot 10$ & $\cdot / V \Delta$ & 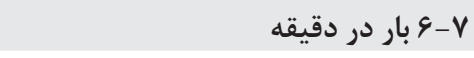 \\
\hline$\cdot / 10$ & $\cdot / \pi \Delta$ & $\cdot 19$ & 1-1 بار در دقيقه \\
\hline . & $\cdot / r$ & $\cdot / r$ & بيش از •ا بار در دقيقه \\
\hline
\end{tabular}




\section{افتثه .}

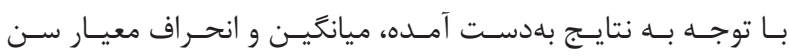

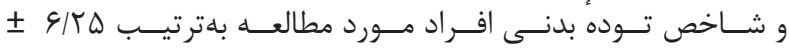

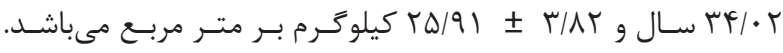

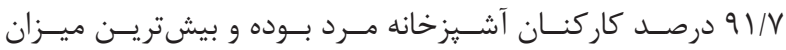

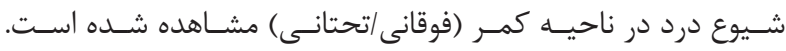

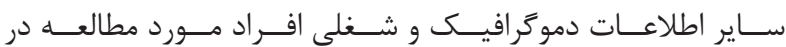

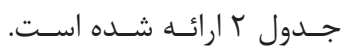

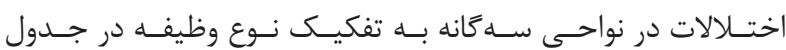

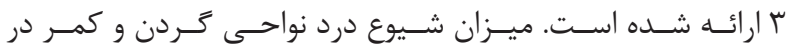

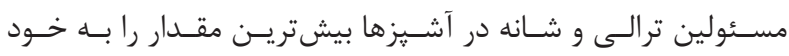
ختصـاص داد.

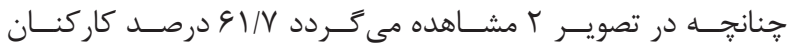

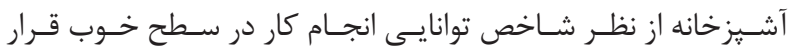
دارنــ.

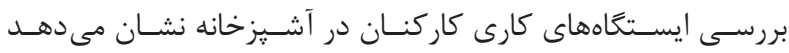

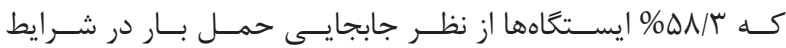

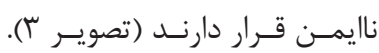

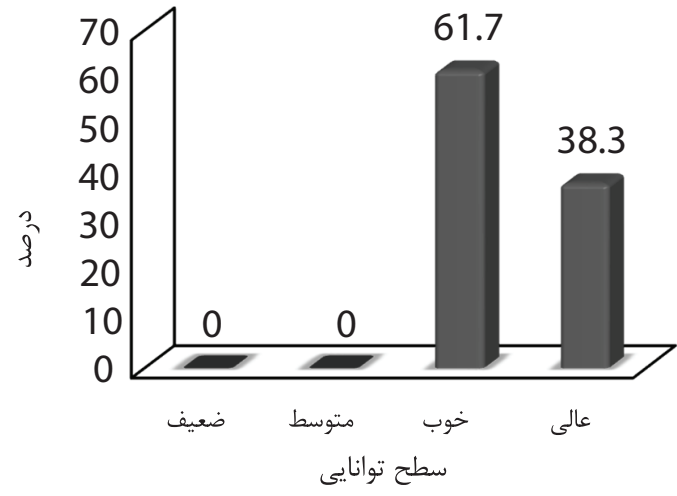

تصوير r: فراوانى نتايج شاخص توانايى انجام كار (•9 = (n)

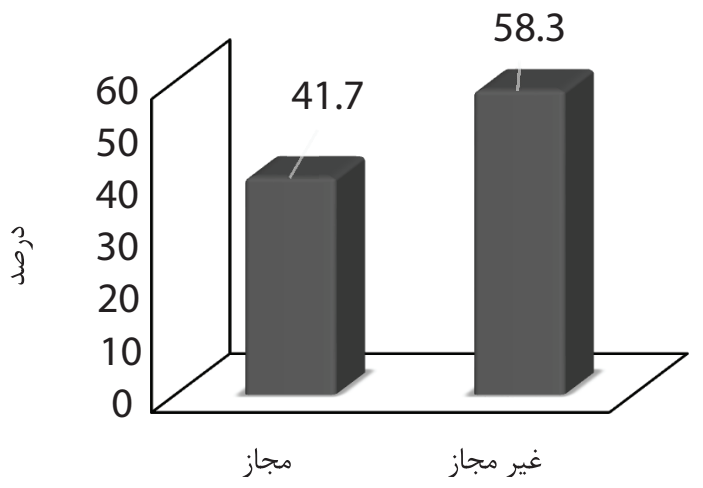

طبقه بندى حمل بار

تصوير ץ: فراوانى نتايج حد مجاز برداشتن بار ( • = (n)
جدول r: اطلاعات دموكر افيك و شغلى افراد مورد مطالعه (•n=9)

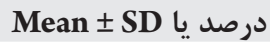
نوع اطلاعات

$\% \Lambda / r$ جنسيت

$\% 91 / \mathrm{V}$ زن

$r / \cdot r \pm \varepsilon / r \Delta$

$V A / 1 T \pm \| / T r$

IVI/OV $\pm V / q$.

$r \Delta / 91 \pm T / A T$

سن، سال

$\% \bowtie \& / Y$

وزن، كيلوكرم سيال

قد، سانتى متر

$\%$ \%r/

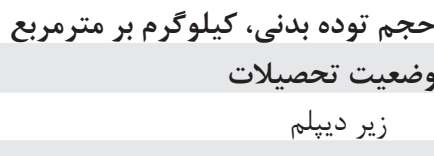

بالاتر ار دييلم.

$q / \uparrow \Delta \pm r / \cdot \Delta$

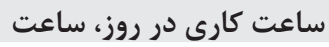
ساعت كارى در روز، ساعت داعت ساعت

$\% \bowtie 9 / V$ ^ ساعت كارى كارى در روز

$\%$ \%r/r بيش از 1 ساعت كارى كارى روز كارى در هفته

$\% \mathrm{~V} / \mathrm{r}$

\%rs/V

צ روز در هفته

V روز در هفته سابقه كار

$\% \& \wedge / r$

كمتر از ه سال

\%r।/9

بيش از ه سال نوع وظيفه

$\% 1$. يخت و يز سرآشيز

$\%$ r.

آشيز

خدماتى اشير

$\%$ \% $/ 4$

كمك آشيز

\%rs/V

مسئول ترالى

\%Fa/D

$\%+\backslash / V$ شيوع درد در نواحى ^ مَانه بدن $\%$ $\%$ rd شانه رنج $\% \vee \wedge / r$ دست/ مج دست $\% 11 / \mathrm{V}$ كمر (فوقانى/تحتانى) دانى $\%$ rq ران زانو

$\% 1$. ساق يّا/ يا

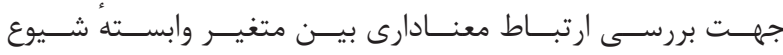

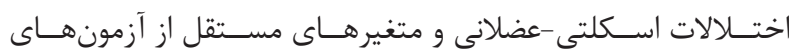

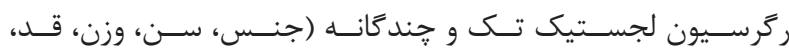

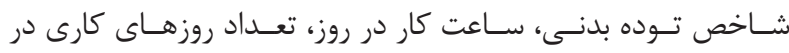

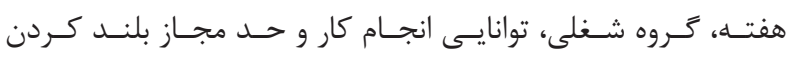

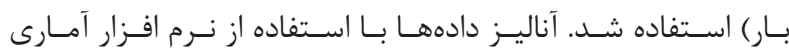
SPSS 


\begin{tabular}{|c|c|c|c|c|c|c|}
\hline \multicolumn{7}{|c|}{ جدول با: ميزان فراوانى شيوع اختلالات اسكلتى-عضلانى در نواحى سهَانه (كردن، شانه و كمر) به تفكيك وظيفه (•"n=9) شيوع } \\
\hline \multicolumn{2}{|c|}{ 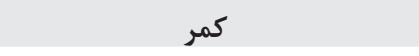 } & \multicolumn{2}{|c|}{ شانه } & \multicolumn{2}{|c|}{ كردن } & \multirow{2}{*}{ نوع وظيفه } \\
\hline خير، تعداد (\%) ( & بله، تعداد (\%) & خير، تعداد (\%) & بله، تعداد (\%) & خير، تعداد (\%) ( & بله، تعداد (\%) & \\
\hline$(r+\mathcal{R}) T$ & $(99 / 9))^{\uparrow}$ & $(99 / 9) r^{\uparrow}$ & $(r \mu / \mathcal{L}) t$ & $(99 / 9) r^{\uparrow}$ & $(r / \mathcal{L}) r$ & 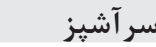 \\
\hline$(r \Delta) r$ & $(\vee \Delta) 9$ & $(F / / 9) \Delta$ & $(\Delta \wedge / \mathcal{F}) \vee$ & $(\Delta \Lambda / \mathcal{L}) V$ & $(\xi / / 9) \Delta$ & آشيز \\
\hline$($ (Tr) 9 & $(V Y) Y$. & $(V+/ 1) 19$ & $(Y \& / 9) \vee$ & $(9) / 9) 19$ & $(r N / \mathcal{F}) 1$. & كمك آشيز \\
\hline$(1 r / \Delta) r$ & $(\Lambda \vee / \Delta) \backslash \mathcal{F}$ & $(F \Gamma / \Lambda) V$ & $(\Delta \varphi / T) q$ & $(\Pi V / \Delta) \varphi$ & $(G Y / \Delta) 1$. & مسئول ترالى \\
\hline
\end{tabular}

\begin{tabular}{|c|c|c|c|c|}
\hline \multicolumn{5}{|c|}{ جدول f: حد مجاز جابجايى بار در بين كاركنان آشيزخانه بر اساس تفكيك نوع وظيفه (•4 = (n) } \\
\hline مسئول ترالى، تعداد (\%) & كمى آشيز، تعداد (\%) & آشيز، تعداد (\%) & سر آشيز، تعداد (\%) & \\
\hline$(\xi / r) 1$ & $(\Delta \cdot) 1 r$ & $(\Delta \cdot)^{9}$ & $(\Lambda \mu / \mu) \Delta$ & مجاز \\
\hline$(9 \mu / \Lambda) 10$ & $(\Delta \cdot) \mid r$ & $(\Delta \cdot) \varphi$ & $(\mid 9 / V) 1$ & غير مجاز \\
\hline
\end{tabular}

\begin{tabular}{|c|c|c|c|c|c|c|}
\hline كمر بر اساس & حى تردن، شانه و & كلتى-عضلانى در نز & | شيوع اختلات | & و و ارخونوميك مؤثر : & رهاى فردى، شغلح & آزمون ركدرل ه: نتايج \\
\hline \multirow[t]{2}{*}{ جندكانه، } & تكىمتغيرى، & "جند & تكىمتغيرى، & 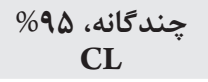 & \multirow[t]{2}{*}{ تكىمتغيرى، } & \multirow[b]{2}{*}{ جنس } \\
\hline & & & & & & \\
\hline 1 & 1 & 1 & 1 & 1 & 1 & 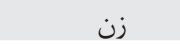 \\
\hline $\begin{array}{c}(\cdot / \cdot \Delta-\varepsilon Y / \Lambda F) \\
1 / \Lambda q\end{array}$ & $\begin{array}{c}(\cdot /|\Delta-| Y / \Delta T) \\
1 / \Delta .\end{array}$ & $\begin{array}{c}(\cdot 1 \cdot 1-\mid N / 4 \varepsilon) \\
\cdot / 4 Y\end{array}$ & $\begin{array}{c}(\cdot|\Delta \Lambda-\Delta r /| l) \\
\Delta / \Delta \varepsilon\end{array}$ & $\begin{array}{c}(\cdot / \cdot V-r|\cdot| \mathcal{E} r) \\
\mathcal{E} / \Lambda\end{array}$ & $\begin{array}{c}(\cdot|\Delta \Lambda-\Delta \mu /| \mid) \\
\Delta / \Delta \varepsilon\end{array}$ & 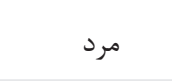 \\
\hline $1 / 19$ & & & & & & سن، سال \\
\hline 1 & 1 & 1 & 1 & 1 & 1 & $\leq r r$ \\
\hline \multirow[t]{2}{*}{$\begin{array}{c}(\cdot / \cdot \varphi-r / V \cdot) \\
\cdot / r \varphi\end{array}$} & $\begin{array}{c}(\cdot / f \mathcal{F}-\mathcal{F} / \mathcal{F} \Delta) \\
1 / \mathcal{F}\end{array}$ & $\begin{array}{c}(\cdot / 11-F / / 4) \\
\cdot / 99\end{array}$ & $\begin{array}{c}(\cdot / T I-Y / 4 \mid) \\
\cdot \mid A Y\end{array}$ & $\begin{array}{c}(\cdot / / F-V / V \cdot) \\
1 / \cdot F\end{array}$ & $\begin{array}{c}(\cdot / T \mid-T /\{\mid) \\
\cdot \mid A V\end{array}$ & $\geq \mu r$ \\
\hline & & & & & & وزن، كيلوگرم \\
\hline 1 & 1 & 1 & 1 & 1 & 1 & $\leq \mathrm{V} \Delta$ \\
\hline \multirow[t]{2}{*}{$\begin{array}{c}(\cdot / \cdot T-Y / 1 \cdot) \\
\cdot / T r\end{array}$} & $\begin{array}{c}(\cdot / \mathcal{Y V}-F / q I) \\
1 / \Delta T\end{array}$ & $\begin{array}{c}(\cdot / \cdot 1-T / T \cdot) \\
\cdot / 1 \Delta\end{array}$ & $\begin{array}{c}(\cdot / 19-1 / \Delta 9) \\
\cdot / \Delta \Delta\end{array}$ & $\begin{array}{c}(\cdot / \cdot \cdot V-r / V q) \\
\cdot / 1 \digamma\end{array}$ & $\begin{array}{c}(\cdot / 19-1 / \Delta 9) \\
\cdot / \Delta \Delta\end{array}$ & 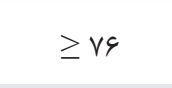 \\
\hline & & & & & & قد، سانتى متر \\
\hline 1 & 1 & 1 & 1 & 1 & 1 & $\leq \mathrm{IVT}$ \\
\hline \multirow[t]{2}{*}{$1 / \pi$} & $\begin{array}{c}(\cdot / T Y-Y / Y Y) \\
\cdot / Y I\end{array}$ & $\begin{array}{c}\left(\cdot / \cdot \mathcal{F}^{-}-r / T r\right) \\
\cdot / T \Delta\end{array}$ & $\begin{array}{c}(\cdot / T F-1 / \wedge \Delta) \\
\cdot / 99\end{array}$ & $\begin{array}{c}(\cdot / \cdot r-\psi / \cdot v) \\
\cdot / r \cdot\end{array}$ & $\begin{array}{c}\left(\cdot / T F^{-}-1 / \Lambda \Delta\right) \\
\cdot / 9 \varphi\end{array}$ & $\geq \operatorname{IV} r$ \\
\hline & & & & & & عاBMI كيلوَّرم بر مترمربع \\
\hline 1 & 1 & 1 & 1 & 1 & 1 & $\leq r \Delta / \varphi$ \\
\hline $\begin{array}{c}(1 / V-r \mid 9 / T) * * \\
\mid r / T r\end{array}$ & $\begin{array}{c}(\cdot / T Y-Y / Y Y) \\
\cdot / V I\end{array}$ & $\begin{array}{c}1 / \Lambda 1 \\
(\cdot / I I-r V / \Lambda \cdot)\end{array}$ & $\begin{array}{c}(q) \cdot-r / 19) \\
1 / 1 q\end{array}$ & $\begin{array}{c}(\cdot / r V-I F V / q F) \\
q / T r\end{array}$ & $\begin{array}{c}(\cdot / 4 \mid-r / 19) \\
1 / 14\end{array}$ & $\geq r \Delta / V$ \\
\hline \multicolumn{7}{|c|}{ ساعت كار در روز، ساعت } \\
\hline 1 & 1 & 1 & 1 & 1 & 1 & $\leq \wedge$ \\
\hline $\begin{array}{c}(\cdot / \cdot V-r / \cdot \Delta) \\
\cdot / F \Lambda\end{array}$ & $\begin{array}{c}(\cdot / r \cdot-r / \cdot 9) \\
\cdot / 9 V\end{array}$ & $\begin{array}{c}(\cdot / \cdot 9-1 / \cdot \Delta) \\
\cdot / \cdot r\end{array}$ & $\begin{array}{c}(\cdot / r q-r / r \cdot) \\
\cdot / A r\end{array}$ & $\begin{array}{c}(\cdot / \cdot \cdot 9-1 / 9 \Lambda) \\
\cdot / 1 r\end{array}$ & $\begin{array}{c}(\cdot / T Q-r / r \cdot) \\
\cdot / A r\end{array}$ & $\geq 9$ \\
\hline \multicolumn{7}{|c|}{ تعداد روزهاى كارى در هفته } \\
\hline 1 & 1 & 1 & 1 & 1 & 1 & (ورز \\
\hline \multirow[t]{2}{*}{$\cdot / \pi r$} & $\begin{array}{c}(\cdot / \mid q-1 / q \Lambda) \\
\cdot / 4 q\end{array}$ & $\begin{array}{c}(\cdot 1 \cdot \cdot 1-r / V q) \\
\cdot 1 \cdot V\end{array}$ & $\begin{array}{c}(\cdot|\Lambda|-\Lambda|q|) \\
r \mid g Y\end{array}$ & $\begin{array}{c}\cdot / T r \\
(\cdot / T \Lambda \cdot / T \Delta)\end{array}$ & $\begin{array}{c}(\cdot|\Lambda|-\Lambda / g \mid) \\
Y / g Y\end{array}$ & V اوز V \\
\hline & & & & & & كروه شغلى \\
\hline 1 & 1 & 1 & 1 & 1 & 1 & آشيز \\
\hline $\begin{array}{c}(\cdot / \cdot-r \mu / T I) \\
\cdot / \cdot r\end{array}$ & $\begin{array}{c}(\cdot 1 \cdot V-\Delta / 9 V) \\
\cdot 194\end{array}$ & $\begin{array}{c}(\cdot / \cdot r-\Delta / \Delta q) \\
\cdot / / r\end{array}$ & $\begin{array}{c}(\cdot / \cdot 9-\Delta / \& \Psi) \\
\cdot / V \cdot\end{array}$ & $\begin{array}{c}(\cdot / \cdot 1-r \Delta / T r) \\
\cdot / \Lambda \cdot\end{array}$ & $\begin{array}{c}(\cdot / \cdot 9-\Delta / \& \mu) \\
\cdot / V \cdot\end{array}$ & سرآشيز \\
\hline $\begin{array}{c}(\cdot|| F-r \cdot / F q) \\
|/ Y|\end{array}$ & $\begin{array}{c}(\cdot / T-Y / 9 T) \\
\cdot / q T\end{array}$ & $\begin{array}{c}(\cdot / \cdot \cdot \Delta-1 / \Delta V) \\
\cdot / \cdot \Lambda\end{array}$ & $\begin{array}{c}(\cdot / r I-r / \Delta T) \\
\cdot / \Lambda Y\end{array}$ & $\begin{array}{c}\text { T/TT } \\
(\cdot / T-H N / I V)\end{array}$ & $\begin{array}{c}(\cdot / r \mid-r / \Delta T) \\
\cdot / A V\end{array}$ & كمك آشيز \\
\hline
\end{tabular}




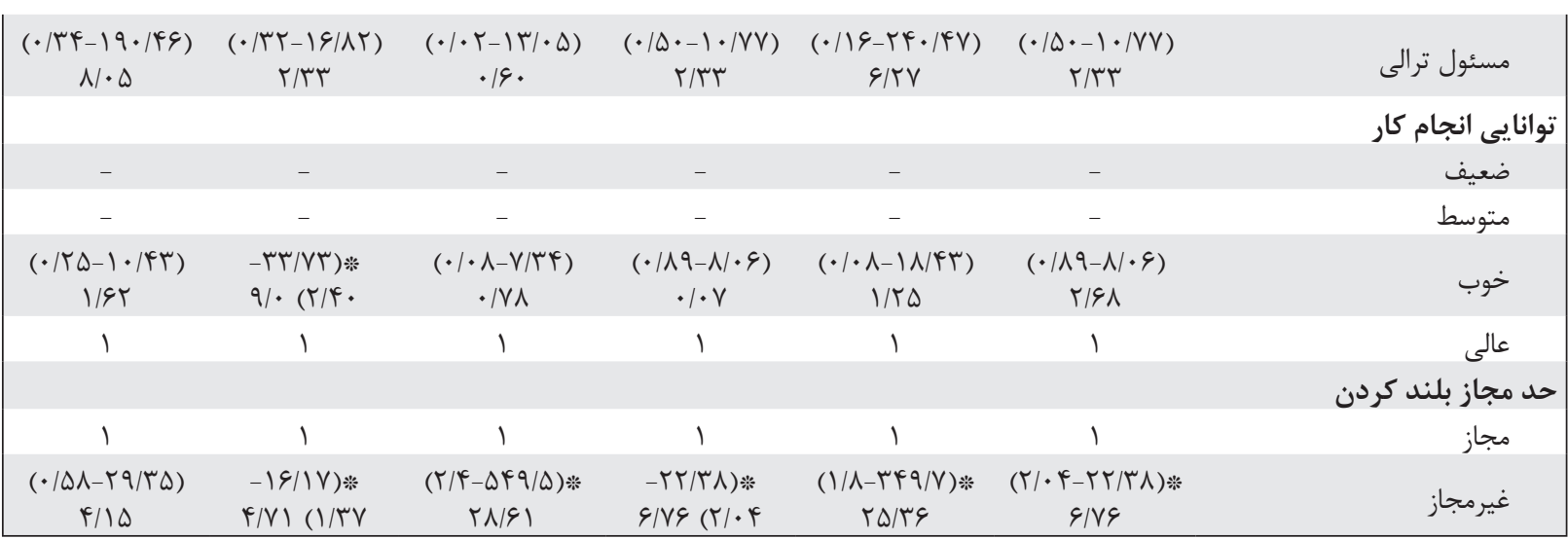

* معنادارى در سطح كمتر از هـ/•

را از عوامـل شـيوع كمــر درد دانســهـاند كــه بــا مطالعـهُ حاضــر

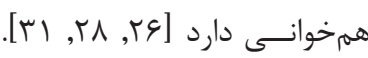

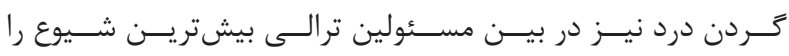

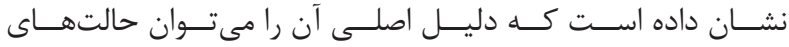

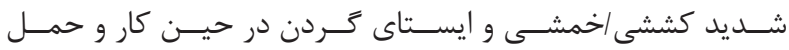

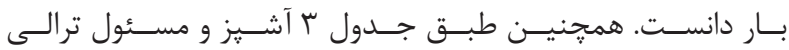

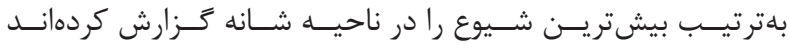

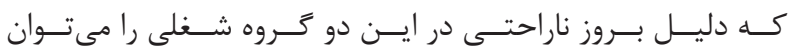

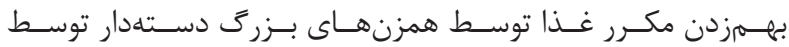

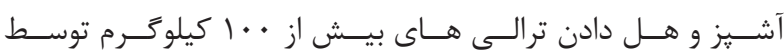

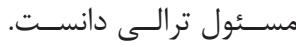

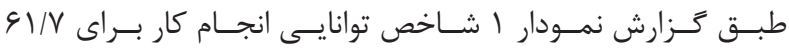

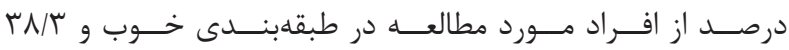

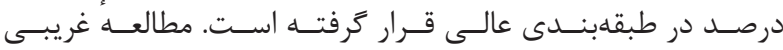

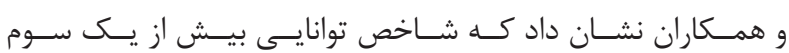

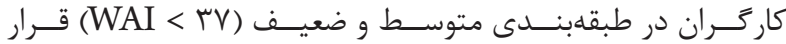

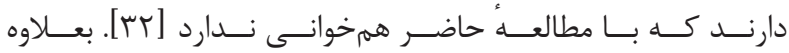
يافتههــاى مطالعــه جوهانسى و همــكاران، نــرخ توانايسى انجــام كار

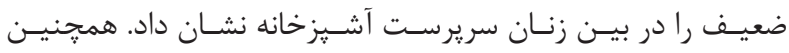

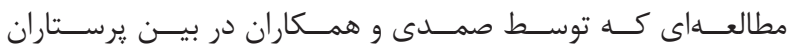

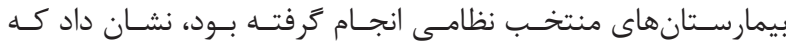

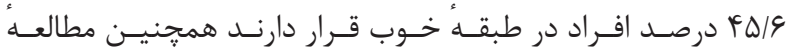

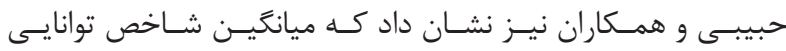

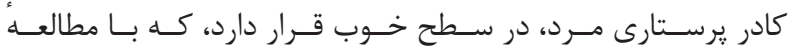

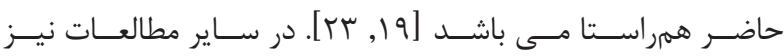

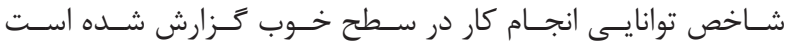

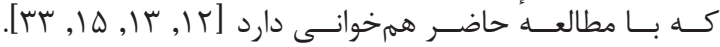

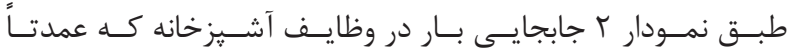

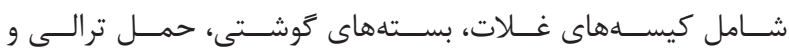

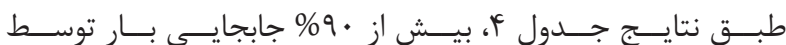

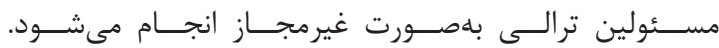

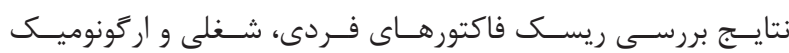

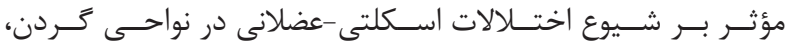

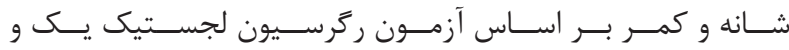

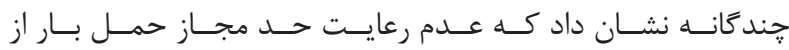

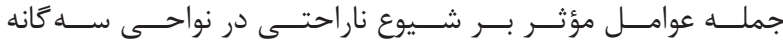

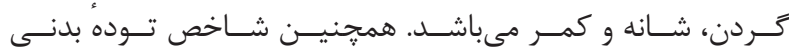

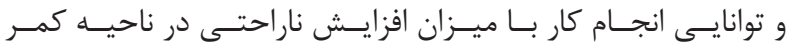
مرتبـــ مى باشـــند (جـــدول ف).

طبـق جــدول rا، بيشتريـن ميـزان شـيوع اختـلالات بلهترتيـب در

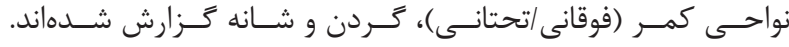

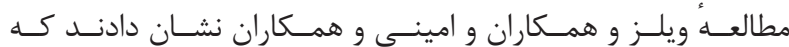

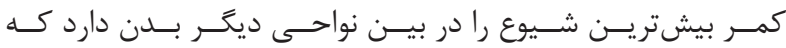

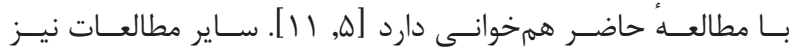

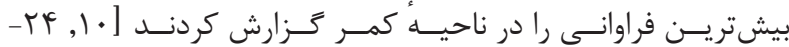

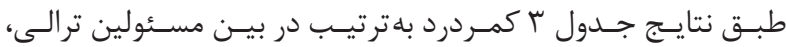

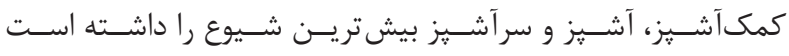

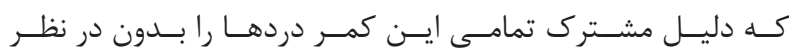

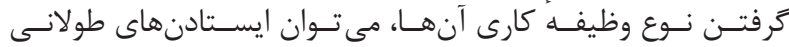

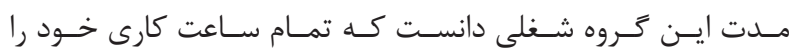

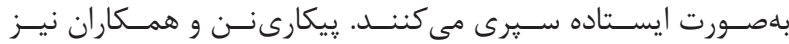

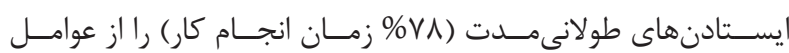

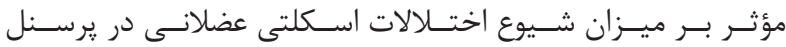

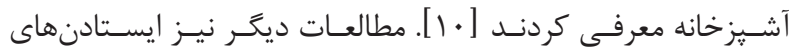

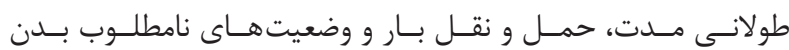




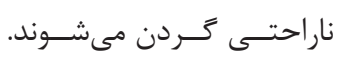

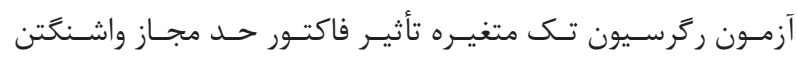

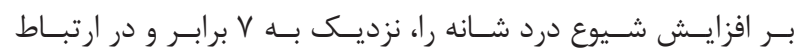

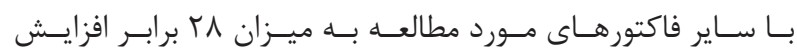

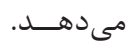

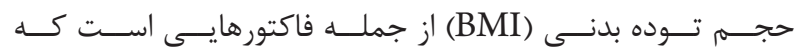

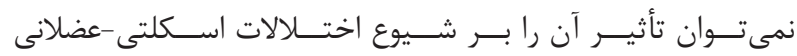

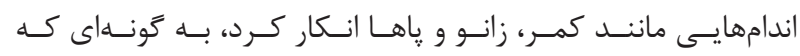

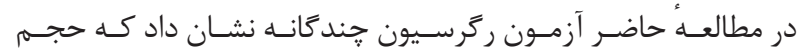

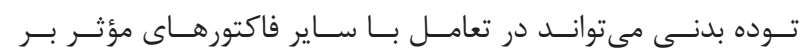

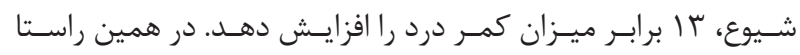

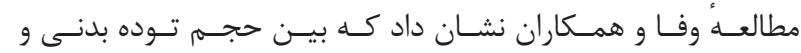

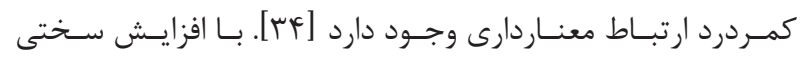

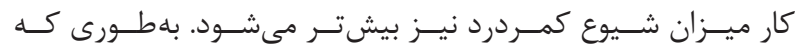

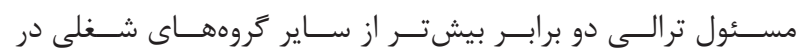

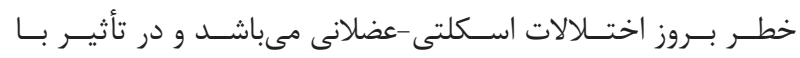

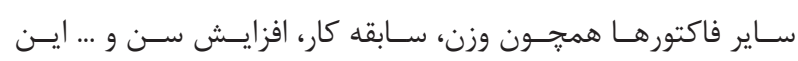

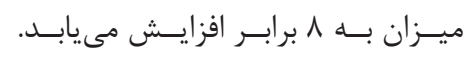

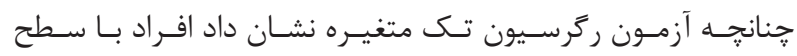

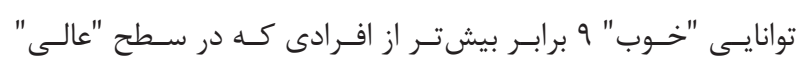

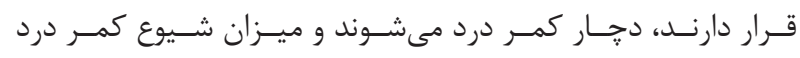

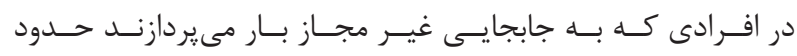

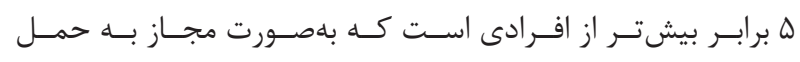

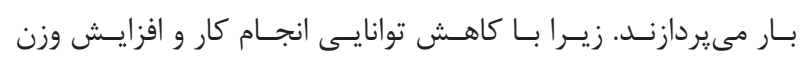

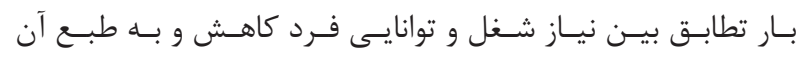

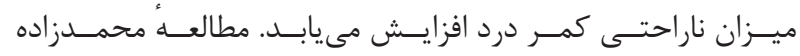

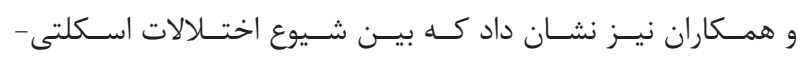

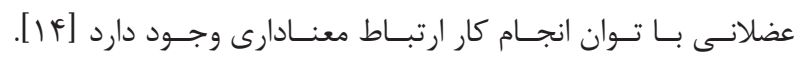

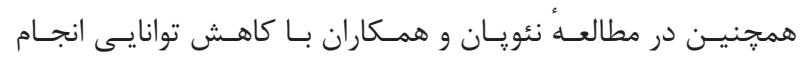

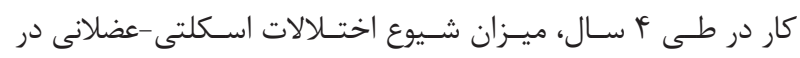

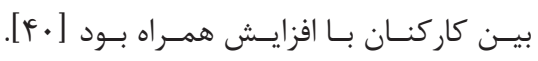

\section{نتيجهكيرى}

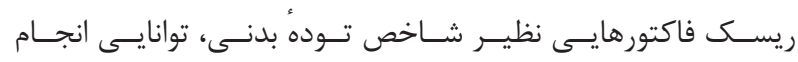

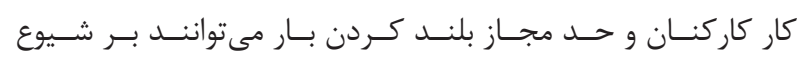

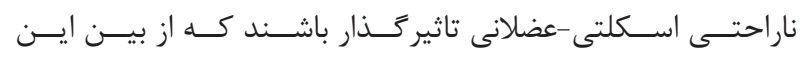

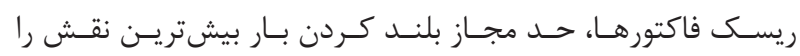

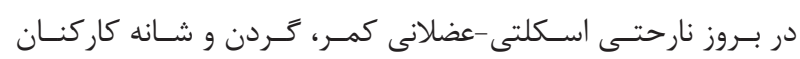

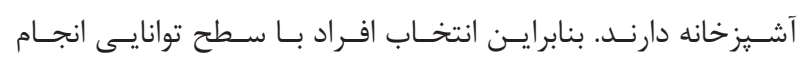

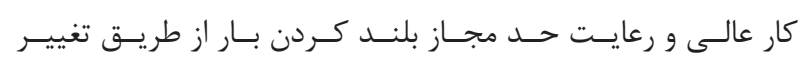

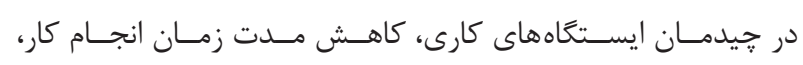

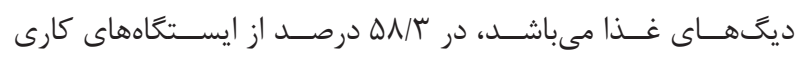

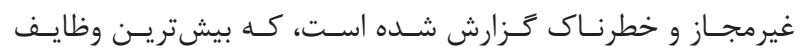

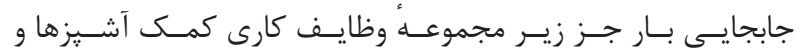

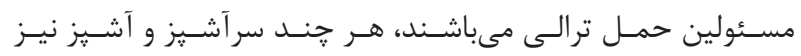

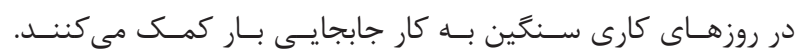

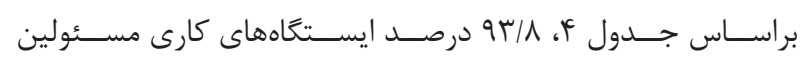

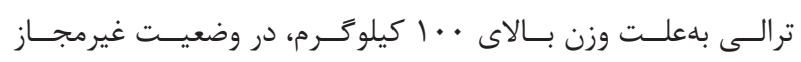

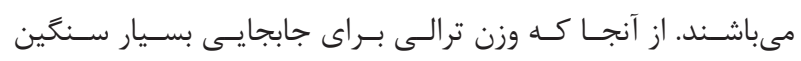

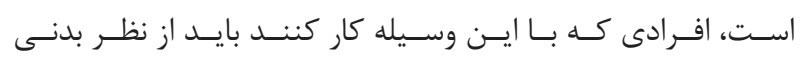

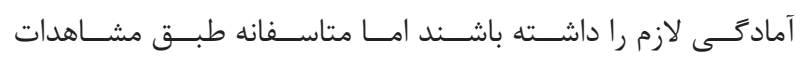

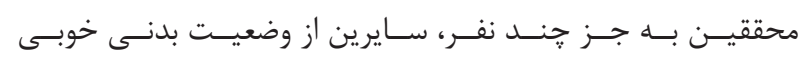

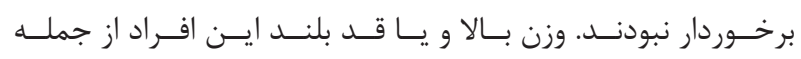

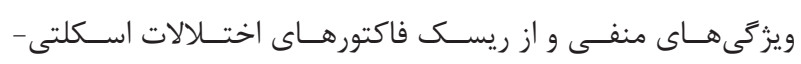

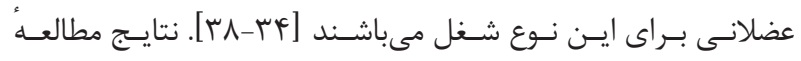

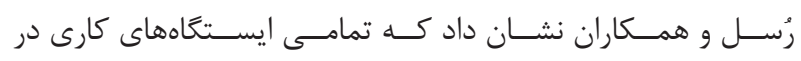

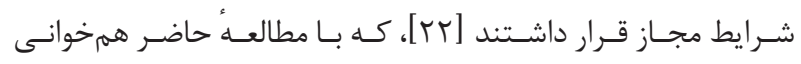

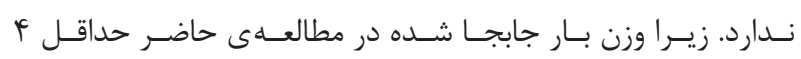

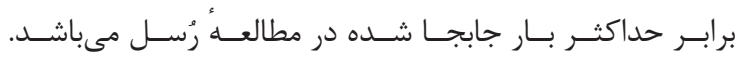

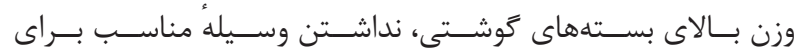

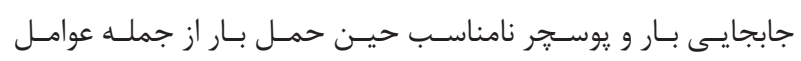

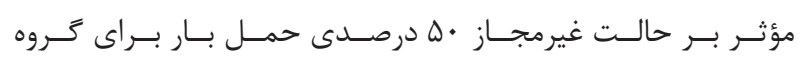

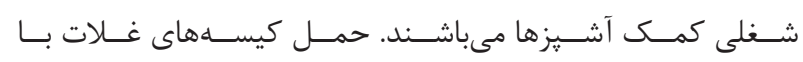

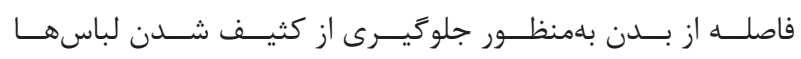

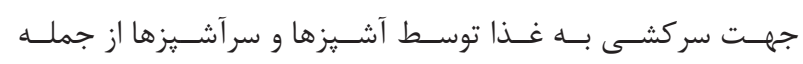

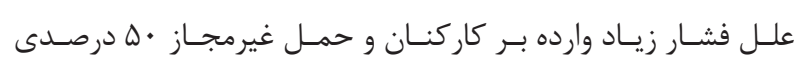

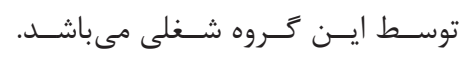

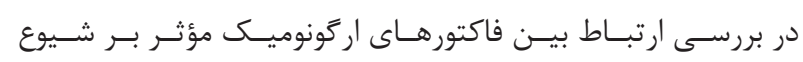

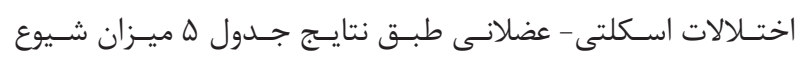

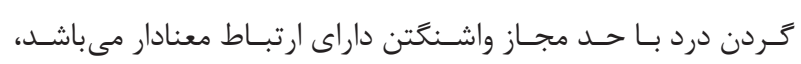

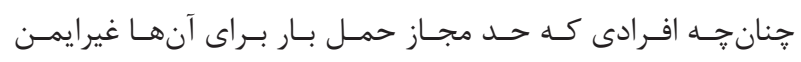

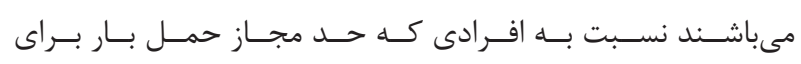

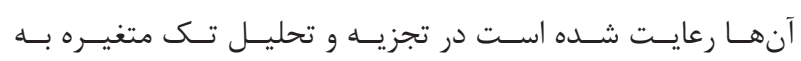

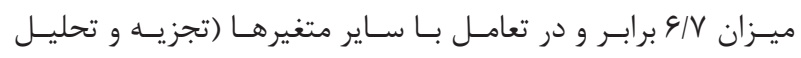

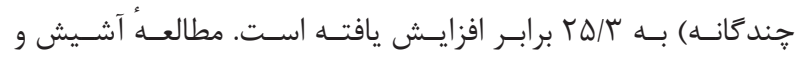

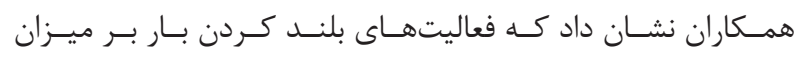

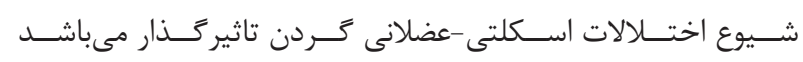

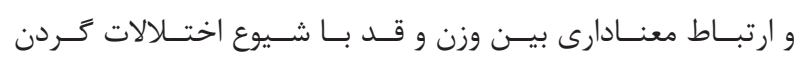

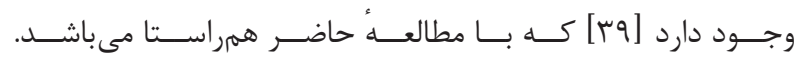

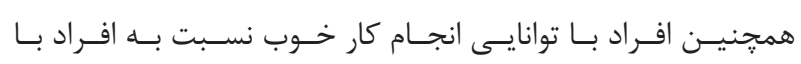

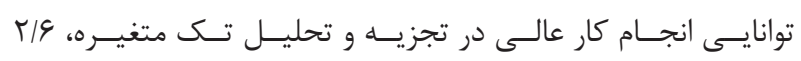

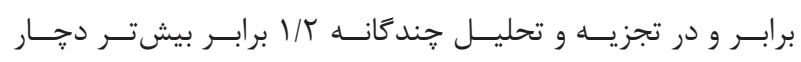




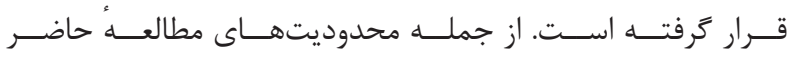

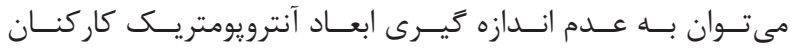

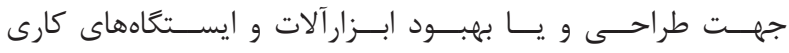
مشـكلدار اشـاره كـــــ

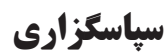

XXX

\section{REFERENCES}

1. Gardfaramarzi R, Dehghani Y, Naeini H, Falahati M, Zokaei M. [Body posture assessment welders posture assessment methods OWAS]. J Occupat Med Special. 2011;3(1):4-9.

2. Gholami A, Soltanzadeh A, Abedini R, Sahranavard M. Ergonomic Assessment of Musculoskeletal Disorders Risk by Rapid Upper Limb Assessment (RULA) Technique in a PorcelainManufacturing Factory. J Rese Health. 2014;4(1):608-12.

3. Setoodeh H, Nazaripoor E, Haghayegh A, Shaban Sarvestani S. Risk Assessment of Musculoskeletal Disorders and Determination of the Associated Factors among Workers of a Dairy Products Factory. J Health Sci Surveill Sys. 2014;2(4):134-9.

4. Mostaghasi M, Davari M, Mollaei F, Salehi M, Mehrparvar A. [The frequency of musculoskeletal disorders and to assess posture while working as RULA in auto parts manufacturing industry workers]. J Occupat Med Special. 2011;3(4):26-32.

5. Amini A, Sazandeh Z, Varmazyar S. [Evaluate musculoskeletal disorders among workers in Qazvin restaurants. 9th National Congress Occupational Health and Safety]. Yazd Univ Med Sci. 2011.

6. Haukka E, Leino-Arjas P, Solovieva S, Ranta R, Viikari-Juntura E, Riihimaki H. Co-occurrence of musculoskeletal pain among female kitchen workers. Int Arch Occup Environ Health. 2006;80(2):141-8. DOI: $10.1007 / \mathrm{s} 00420-006-0113-8$ PMID: 16688464

7. Haukka E, Leino-Arjas P, Ojajarvi A, Takala EP, Viikari-Juntura E, Riihimaki H. Mental stress and psychosocial factors at work in relation to multiple-site musculoskeletal pain: a longitudinal study of kitchen workers. Eur J Pain. 2011;15(4):432-8. DOI: 10.1016/j. ejpain.2010.09.005 PMID: 20932789

8. Chim J, editor Ergonomics Workload Analysis For The Prevention of Musculoskeletal Disorders in Food Services in The Health Sector. Human Factors and Ergonomics Society of Australia 42nd Annual Conference; 2006; Australia

9. Jafari M, Fazli B, Noorani M, Sharifpour Z, Gard Faramarzi R. [Risk assessment of musculoskeletal disorders with RULA method and survey effect of ergonomic training on working conditions Khyatayan]. J Occupat Med Special. 2013;5(2):43-50.

10. Ehlers CL, Liu W, Wills DN, Crews FT. Periadolescent ethanol vapor exposure persistently reduces measures of hippocampal neurogenesis that are associated with behavioral outcomes in adulthood. Neuroscience. 2013;244:1-15. DOI: 10.1016/j.neuroscience.2013.03.058 PMID: 23567812

11. Poyakian M, Zakerian SA, Avakh A, Mohamadian F, Kangavari M. Worker's work ability index in the fruit and vegetable stands in Tehran in 2014. Pajouhan Sci J. 2015;13(4):34-41.

12. Hajizadeh F, Motamedzade M, Golmohammadi R, Soltanian A. Work ability assessment and its relationship with severity of musculoskeletal disorders among workers in a cement plant. J Occupat Hygiene Engin. 2015;2(2):15-22.

13. Mohammadzadeh M, Habibi E, Hasanzadeh A. [Relationship between work ability and mental workload with musculoskeletal disorders in industrial jobs]. J Prevent Med. 2015;2(4):29-38.

14. Eyvazlou M, Mazloumi A, Farshad A, Hoseini F. [Analytical evaluation of work ability index and its determining factors among workers of a car manufacturing industry]. Iran Occupat Health. 2012;9(2):40-9.

15. Alavinia S, Hosseini S. [WAI important tool in health professional and occupational medicine To identify workers at risk of premature exit from the workplace]. J North Khorasan Univ Med Sci. 2011;3(2):49-53.

16. Ilmarinen J, Tuomi K, Klockars M. Changes in the work ability of

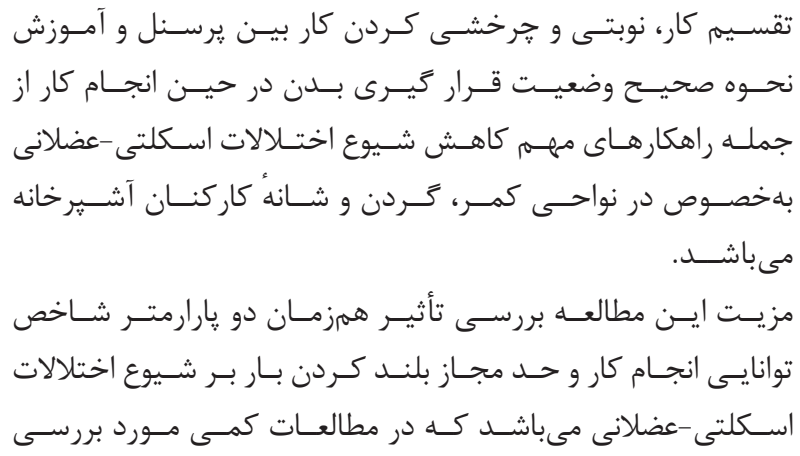

active employees over an 11-year period. Scand J Work Environ Health. 1997;23 Suppl 1:49-57. PMID: 9247995

17. Celedova L, Babkova K, Rogalewicz V, Cevela R. The Work Ability Index for persons aged 50+ as an instrument for implementing the concept of Age Management. Contact. 2014;16(4):e242-e8. DOI: 10.1016/j.kontakt.2014.10.003

18. Samadi S, Golmohammadi A. [Study the Relation between Occupational Stress and Work Ability Index among Nurses of Selected Military Hospital]. J Police Med. 2013;2(4):223-3.

19. Haukka E, Ojajarvi A, Takala EP, Viikari-Juntura E, Leino-Arjas P. Physical workload, leisure-time physical activity, obesity and smoking as predictors of multisite musculoskeletal pain. A 2-year prospective study of kitchen workers. Occup Environ Med. 2012;69(7):485-92. DOI: 10.1136/oemed-2011-100453 PMID: 22539656

20. Keikha-Moghaddam A. [Ergonomics Assesment Methods Selection and Application Guide]. Iran: Fanavaran; 2012.

21. Russell SJ, Winnemuller L, Camp JE, Johnson PW. Comparing the results of five lifting analysis tools. Appl Ergon. 2007;38(1):91-7. DOI: 10.1016/j.apergo.2005.12.006 PMID: 16867298

22. Habibi E, Dehgan H, Zeinodini M, Yousefi H, Hasanzadeh A. [The Relation between Work Ability Index and Physical Work Capacity based on Fox Equation for VO2 Max in Male Nursing Staff of Isfahan Hospitals, Iran]. J Health Sys Res. 2011;7(5):606-14.

23. Pekkarinen A, Anttonen $H$. The effect of working height on the loading of the muscular and skeletal systems in the kitchens of workplace canteens. Appl Ergon. 1988;19(4):306-8. PMID: 15676673

24. Choobineh A, Rahimifar H, Jahangiri M, Mahmoodkhani S. [Musculoskeletal injuries and their associated risk factors in office workplaces]. Iran Occupat Health. 2011;8(4):70-81.

25. Choobineh A, Mokhtarzadeh A, Salehi M, Tabatabaei S. [Ergonomic evaluation of exposure to musculoskeletal disorders risk factors by QEC technique in a rubber factory]. Med J. 2008;7(1):46-55.

26. Fallah H, Koohnavard B, Cheraghi M, Yousefi M, editors. [Evaluation of ergonomic to quickly assess exposure method in the dining hall workers of the University of Medical Sciences in 2014]. 1st Biennial Iranian Conference on Ergonomics; Hamedan University of Medical Sciense; 2014; Hamedan

27. Kohansal S, Kohpaei R, Gharlipour Z, Habibi P, Ziaei M, Gilasi HR, et al. [Ergonomic Evaluation of Musculoskeletal Disorders Among Kitchen Workers by QEC Technique in the Tehran University of Medical Sciences]. Sci J Ilam Univ Med Sci. 2013;20(4):19-29.

28. Mehrparvar A, Ranjbar S, Mostaghasi M, Salehi M. [Assess the risk of musculoskeletal disorders by QEC method in a food factory]. 2011;3(2):54-60.

29. Sarsangi V, Matlabi M, Fallah H, Zarei E, Khajevandi A, Saghi M. [Detection and risk assessment of musculoskeletal disorders among the staffs employed in a dish manufacturing company using the QEC method and Nordic questionnaire]. Q J Sabzevar Univ Med Sci. 2014;20(5):706-15.

30. Chyuan JY, Du CL, Yeh WY, Li CY. Musculoskeletal disorders in hotel restaurant workers. Occup Med (Lond). 2004;54(1):55-7. PMID: $\underline{14963256}$

31. Abedini R, Choobineh A, Soltanzadeh A, Ghiasvand R, KazemHaghighi M. [Ergonomic evaluation of exposure to musculoskeletal disorders risk factors by Quick Exposure Check (QEC) technique in a metal structure manufacturing factory]. J Health Sci Jundishapoor. $2012 ; 4(2): 13-20$.

32. Gharibi V, Mokarami H, Taban A, Yazdani Aval M, Samimi K, Salesi 
M. Effects of Work-Related Stress on Work Ability Index among Iranian Workers. Saf Health Work. 2016;7(1):43-8. DOI: 10.1016/j. shaw.2015.10.005 PMID: 27014490

33. Koohpayezadeh J, Mokamelkhah E, Alavinia M, Karimi Farshi L, Akbari F. [Study on predictive value of work ability index to predict sick leave and disability caused by work]. Razi J Med Sci. 2014;21(125):126-30.

34. Bawab W, Ismail K, Awada S, Rachidi S, Hajje A, Salameh P. Prevalence and Risk Factors of Low Back Pain among Office Workers in Lebanon. Int J Occupat Hygiene. 2015;7(1):45-52.

35. Hershkovich O, Friedlander A, Gordon B, Arzi H, Derazne E, Tzur D, et al. Associations of body mass index and body height with low back pain in 829,791 adolescents. Am J Epidemiol. 2013;178(4):603-9. DOI: $10.1093 /$ aje/kwt019 PMID: 23690249

36. Heuch I, Heuch I, Hagen K, Zwart JA. Association between body height and chronic low back pain: a follow-up in the Nord-Trondelag
Health Study. BMJ Open. 2015;5(6):e006983. DOI: 10.1136/bmjopen-2014-006983 PMID: 26078308

37. Nagasu M, Sakai K, Ito A, Tomita S, Temmyo Y, Ueno M, et al Prevalence and risk factors for low back pain among professional cooks working in school lunch services. BMC Public Health. 2007;7:171. DOI: 10.1186/1471-2458-7-171 PMID: 17650300

38. Sadeghi N, Habbibi E. [The relationship between musculoskeletal disorders with anthropometric indices among bus drivers in Isfahan]. Iran Occupat Health J. 2009;6(1):6-15.

39. Nimbarte AD. Risk of neck musculoskeletal disorders among males and females in lifting exertions. Int $\mathrm{J}$ Indust Ergonom. 2014;44(2):253-9. DOI: 10.1016/j.ergon.2013.01.007

40. Neupane S, Miranda H, Virtanen P, Siukola A, Nygard CH. Multi-site pain and work ability among an industrial population. Occup Med (Lond). 2011;61(8):563-9. DOI: 10.1093/occmed/kqr130 PMID: $\underline{21846813}$ 


\title{
Investigation of Correlation of Musculoskeletal Disorders With Work Ability Index and Allowable Load Lifting Limit
}

\author{
Seyed Sajad Ataei ${ }^{1}$, Payam Heydari ${ }^{2}$, Sakineh Varmazyar ${ }^{3,}{ }^{*}$ \\ ${ }^{1}$ BSc of Occupational Health Engineering, School of Public Health, Qazvin University \\ of Medical Sciences, Qazvin, Iran \\ ${ }^{2}$ MSc Student in Occupational Health Engineering, School of Public Health, Qazvin \\ University of Medical Sciences, Qazvin, Iran \\ ${ }^{3}$ Assistant Professor, Department of Occupational Health Engineering, Faculty of \\ Health, Qazvin University of Medical Sciences, Qazvin, Iran \\ * Corresponding author: Sakineh Varmazyar, Assistant Professor, Department of Occu- \\ pational Health Engineering, Faculty of Health, Qazvin University of Medical Sciences, \\ Qazvin, Iran.E-mail:svarmazyar@qums.ac.ir
}

DOI: 10.21859 /joe- 04043

Received: $21 / 06 / 2016$

Accepted: 02/08/2016

\section{Keywords:}

Musculoskeletal Disorders

Work Ability Index

Lifting

How to Cite this Article:

Ataei S, Heydari P, Varmazyar S. Investigation of Correlation of musculoskeletal Disorders With Work Ability Index and Allowable Load Lifting Limit. JErgo. 2017;4(4):1423. DOI: $10.21859 /$ joe- 04043

(C) 2017 Hamedan University of Medical Sciences.

\begin{abstract}
Introduction: Heavy load lifting or occupational and individual factors are effective hazard agents in the occurrence of musculoskeletal disorders. Therefore, the aim of the present study was investigating the correlation of musculoskeletal disorders with work ability index and allowable load lifting limit.

Methods: This analytical and cross-sectional study was conducted using census $(n=60)$ among the kitchen workers in Qazvin teaching hospitals in 2016. Data were collected through questionnaires including pain prevalence, work ability index and allowable load lifting limit. Data was analyzed by single and multiple regression logistic using SPSS 16 software.

Results: The highest prevalence was observed in the lower back (\%78.3). Of the workers, $\% 61.7$ reported a good level of work ability index and \%58.3 of the workplaces were in unsafe conditions of load handling. Unsafe condition alone increased the prevalence of shoulder and neck pain about 7 times and the lower back pain 4 times, and in interaction with other risk factors, the prevalence of neck and shoulder pains had 25 and 28 times increase, respectively. Also, subjects with good levels of ability compared to subjects with excellent levels of ability were 9 times more in exposure of musculoskeletal disorders.

Conclusions: Risk factors such as body mass index, work ability and allowable load lifting limit can affect the prevalence of musculoskeletal disorders. So, selection of subjects with good and excellent work ability levels and compliance with the load limit are the effective solutions in increasing the productivity and reducing musculoskeletal disorders among kitchen workers.
\end{abstract}

